\title{
Successful Outcomes at Midterm Follow-up of Periacetabular Osteotomy Done for Mild Hip Dysplasia
}

\author{
Rafael J. Sierra, MD ${ }^{1} \quad$ G. David Potter, MD $^{1} \quad$ Eduardo N. Novais, MD ${ }^{2,3}$ Robert T. Trousdale, MD ${ }^{1}$ \\ ${ }^{1}$ Department of Orthopedic Surgery, Mayo Clinic, Rochester, \\ Minnesota \\ ${ }^{2}$ Child and Young Adult Hip Preservation, Boston Children's Hospital, \\ Boston, Massachusetts \\ ${ }^{3}$ Assistant Professor, Harvard Medical School, Boston, Massachusetts \\ Address for correspondence Rafael J. Sierra, MD, Department of \\ Orthopedic Surgery, Mayo Clinic, 200 First St SW, Rochester, \\ MN 55905 (e-mail: sierra.rafael@mayo.edu).
}

J Hip Surg 2017;1:167-172.

\begin{abstract}
Keywords

- hip dysplasia

- periacetabular osteotomy

- hip arthroscopy

- young hip pathology

Young hip surgeons are often faced with the decision to either perform arthroscopic surgery or a periacetabular osteotomy (PAO) in patients with symptomatic mild hip dysplasia (MHD). There is, however, a paucity of data on the results of PAO in this group. The aim of this paper is to report the results of PAOs in patients with MHD and compare those to hips with more severe forms of hip dysplasia (SHD). This data can then be used to compare emerging data reporting the results of hip arthroscopy for MHD. From January, 1996 to May, 2009, 299 hips in 268 patients were identified that underwent $\mathrm{PAO}$ at one institution. After removing those with $<2$ years of follow-up, 182 hips were followed up. The average age of the cohort was 31 years, and $85 \%$ were female. Nineteen hips with lateral center edge (LCE) angle from 18 to $25^{\circ}$ and a Tönnis angle (TA) between 10 and $15^{\circ}$ were considered to have MHD. This group was compared with the rest of the cohort (SHD). The mean clinical follow-up for the MHD group was 121 months. There was no significant difference in demographic variables between the groups. There were no complications in the MHD cohort. Surgical correction resulted in significant improvements in all radiographic measurements consistent with hip dysplasia in both groups. The Harris Hip Score (HHS) improved significantly in both groups ([MHD: 52-92] [SHD: 66-89]). Two hips (10.5\%) in the MHD group and 15 hips (9.2\%) in the SHD group underwent future THA $(p=0.69)$. The survivorship free from THA was $100 \%$, $100 \%$, and $86 \%$ at 3,5 , and 10 years, respectively, in the MHD group. The corresponding rates for hips in the control group at 3, 5, and 10 years, respectively, were $99 \%, 95 \%$, and $81 \%$. PAO in patients with MHD provides predictable improvements in pain, function, and results that are durable and comparable to hips with SHD. This data should be used to compare the early and midterm results of arthroscopic surgery performed in mildly dysplastic hips.
\end{abstract}

Hip dysplasia is a structural abnormality of the pelvis and the proximal femur. The structural abnormality may lead to increased contact stresses around the superolateral aspect of the acetabulum and can lead to labral damage and cartilage degeneration. ${ }^{1-5}$ More severe forms of hip dysplasia (SHD) have been associated with progression to arthritis and the need for total hip arthroplasty (THA). ${ }^{6}$

received

August 30, 2017

accepted after revision

December 21, 2017

published online

March 19, 2018
Traditionally, hip dysplasia has been classified according to the degree of anterior and lateral coverage and the degree of slope of the acetabulum..$^{7-9}$ Using the lateral center edge (LCE) and anterior center edge (ACE) angle: an angle between 20 and 25 degrees is considered to be borderline or represent mild hip dysplasia (MHD). ${ }^{8}$ Furthermore, using the roof or Tönnis angle (TA), a mildly dysplastic or borderline hip is

Copyright $\odot 2017$ by Thieme Medical Publishers, Inc., 333 Seventh Avenue, New York, NY 10001, USA. Tel: +1(212) 584-4662.
DOI https://doi.org/ 10.1055/s-0038-1635102. ISSN 2472-8446. 
considered to have a slope between 10 and 15 degrees. $^{9}$ These milder forms of hip dysplasia may not require treatment if asymptomatic. Murphy et al, in their classic report on the natural history of dysplasia, noted that THA may not be required in this group until after the age of $65 .{ }^{6}$ In practice, however, there are increasing number of patients that present with symptoms attributed to labral and cartilage pathology and instability in the setting of radiographic MHD.

There has been debate as to what the best surgical treatment should be for symptomatic MHD. Proponents of hip arthroscopy for the treatment of this problem argue that the intra-articular pathology can be adequately assessed and treated with hip arthroscopy, and as long as the labrum is not resected, the labrum should heal and restore its function within the hip joint. ${ }^{10}$ Hip arthroscopy alone, however, does not correct the underlying structural abnormality that has led to the labral and cartilage pathology or to address the potential instability, and if the structural abnormality is ignored, symptoms may recur or lead to more problems. ${ }^{11-13}$ Furthermore, proponents of hip arthroscopy argue that correction of the minor structural abnormality through a periacetabular osteotomy (PAO) has increased morbidity and that the recovery of such procedure is longer than that of a hip arthroscopy. ${ }^{14}$ The PAO has certain advantages in this setting that should be noted. Correction of the structural abnormality stabilizes the hip joint and in turn will off load the labrum, protect the articular cartilage, and if the hip center is medialized will improve the mechanics of the hip. The PAO has the potential of improving the long-term prognosis of the native hip joint. ${ }^{15-21}$

To the authors knowledge, to date there are no studies comparing the results of hip arthroscopy and PAO in patients with MHD. Because of the increased number of arthroscopic procedures done in this population, the authors believe that it would be pertinent to review the results of PAO in patients with MHD to serve as comparison to the results obtained with hip arthroscopy done in this patient population. The aim of this paper therefore is to report the clinical results of PAO for treatment of MHD, to evaluate the radiographic correction of the hip dysplasia after the PAO in this group, and to assess the survivorship of the PAO with special attention to conversion to THA when compared with patients with more SHD.

\section{Materials and Methods}

After institutional review board (IRB) approval, all abductor sparing PAO performed between January, 1996 and May, 2009 were reviewed. This comprises a single institution series performed by three surgeons (two authors: R. J. S. and R. T. T.). During the study period, 299 hips in 268 patients underwent PAO for all forms of hip dysplasia. Indications for PAO included patients with symptomatic hip dysplasia and instability that had failed nonoperative management. No patients were excluded from this study on the basis of diagnosis only; therefore, independent of underlying cause of the dysplasia, all patients that underwent PAO were included. During the first 10 years of the study, there were no surgeons in the authors' institution that were performing hip arthroscopy. Between 2007 and 2009, one of the authors performed hip arthroscopy, but none were in patients with hip dysplasia. Even after 2009, the number of hip arthroscopy performed in patients with hip dysplasia was small $(n=20)$ according to the institutions' hip arthroscopy registry. Patients who did not have at least 2 years of clinical follow-up were removed (116 SHD and 1 MHD). There were 182 remaining hips. Patients who underwent a THA were removed from follow-up at the date of that procedure. All preoperative radiographs were reviewed by one of the authors (G. D. P.) after appropriate training was given by one of treating surgeons (R. J. S.). All preoperative radiographs were assessed for measurements consistent with hip dysplasia and included an $\mathrm{LCE}^{8}$ an $\mathrm{ACE},{ }^{7}$ and $\mathrm{TAs}^{9}{ }^{9}$ Additional measurements included assessment of the medial clear space and Tönnis arthritis grade. ${ }^{22}$ MHD was defined as a TA between 10 and 15 degrees and an LCE angle between 18 and 25 degrees. More SHD included TA $>15^{\circ}$ and an LCE angle $<18^{\circ}$. Nineteen patients (19 hips) met the criteria for MHD, and these were compared with a control group of 163 hips that had SHD. Demographic variables in both groups are shown in - Table 1.

The mean age of patients in the MHD group was 31 years (range 12-56) compared with 30 (range 17-44) in the control group. The mean follow-up for the MHD group was 121 months (range 24-236), and the discrepancy in the follow-up has to do with the fact that patients in this group were contacted specifically for this study. There was only one male in the MHD group. One hip of the 19 in the MHD group had undergone a previous hip arthroscopy. Intraoperative and postoperative complications were noted from operative reports and postoperative clinical notes. Clinical outcomes were assessed using the Harris Hip Score (HHS) at the final follow-up. No other clinical outcome measures were obtained preoperatively as we started collecting University of California, Los Angeles (UCLA) activity scores, Marx activity scores, and Hip Disability and Osteoarthritis Scores (HOOS) in 2008. Ninety four percent of the postoperative radiographs were available and were reviewed for improvements in femoral head coverage and special emphasis on progression of hip arthritis as measured by Tönnis grade. Survivorship free from THA was assessed using Kaplan-Meier survivorship curves.

Statistical analysis methods included the use of paired $t$-tests to evaluate the pre- to postoperative changes in radiographic measurements as well as the pre- to postoperative changes in HHSs. Two-sample t-tests were used to compare

Table 1 Patient demographic

\begin{tabular}{|l|l|l|l|}
\hline & $\begin{array}{l}\text { Mean age } \\
\text { (years) }\end{array}$ & $\begin{array}{l}\text { Mean } \\
\text { follow-up } \\
\text { (months) }\end{array}$ & Males \\
\hline $\begin{array}{l}\text { Mild dysplasia } \\
(N=19)\end{array}$ & $\begin{array}{l}31 \\
(12-56)\end{array}$ & $\begin{array}{l}121 \\
(24-236)\end{array}$ & 1 \\
\hline $\begin{array}{l}\text { More severe } \\
\text { dysplasia }(N=163)\end{array}$ & $\begin{array}{l}30 \\
(17-44)\end{array}$ & $\begin{array}{l}50 \\
(24-194)\end{array}$ & 58 \\
\hline
\end{tabular}


the postoperative HHSs between the two cohorts. Survivorship free of THA was calculated using the Kaplan-Meier method; the event-free rates were reported with their corresponding 95\% confidence intervals (CI). All statistical tests were two-sided, and the threshold statistical significance was set at $p<0.05$. All analysis was conducted using SAS version 9.3 (SAS Institute Inc.).

\section{Results}

Nineteen patients in the MHD were followed up; two of whom underwent eventual THA. There were no surgical complications noted in the MHD group. In both groups, surgical treatment of hip dysplasia with PAO improved radiographic coverage of the femoral head (-Table $\mathbf{2}$ ).

The MHD group had improvements in the TA from an average of 11 to 5 degrees $(p<0.001)$ and the LCE angle improved from 21 to 31 degrees $(p<0.001)$. The SHD group also had significant improvements in TA from average 25 to 12 degrees $(p<0.001)$ and an improvement in LCE angle from 5 to 23 degrees $(p<0.001)$. In the MHD group, the Tönnis grade did not progress in 16 hips. One hip progressed from a Tönnis grade of 1 to a 2 and the other hip progressed from a Tönnis grade 2 to a 3 and was one of the eventual patients in the MHD group who underwent a THA. - Fig. 1 is anteroposterior and lateral radiographs of the patient in the MHD group who had Tönnis grade progression and underwent a THA 6 years after a PAO.

The preoperative HHS in patients with MHD improved from 52 to 92 postoperatively ( $p<0.001$ ). Of the 17 hips that did not have a THA or lost to follow-up, good to excellent results were seen in 15 of 17 hips (70 or less = poor; 71-79 = fair; $80-89=$ good; and $>90=$ excellent). Patients with SHD improved from a preoperative HHS of 66 to a postoperative hip score of $89(p<0.001)$. There was no significant difference in postoperative HHS between the two groups ( $p=0.400)$.

The 10-year survivorship of PAO was not significantly different between the two groups. Only two hips in the mild DDH required a THA, one at 6 years (noted above), the other at 15 years. The patient that underwent a THA at 6 years was noted to have a Tönnis arthritis grade of 2 prior to the PAO and eventually had progression in arthritis. (-Fig. 2).

The patient who underwent a THA at 15 years continued to have pain after the THA and was found to have a lumbar radiculopathy that seemed to be a cause of her pain. This patient did not have radiographic progression of the arthritis prior to the THA. The survivorship free from THA was 100\% (95\% CI: [100.0, 100.0]), 100\% (95\% CI: [100.0, 100.0]), 86\% $(95 \% \mathrm{CI}:[63.3,100.0])$ at 3,5 , and 10 years, respectively, in the

Table 2 Radiographic findings

\begin{tabular}{|l|l|l|l|l|l|}
\hline & $\begin{array}{l}\text { Tönnis angle } \\
\text { (pre } \rightarrow \text { post) }\end{array}$ & $\begin{array}{l}\text { Lateral center } \\
\text { edge angle } \\
\text { (pre } \rightarrow \text { post) }\end{array}$ & $\begin{array}{l}\text { Anterior center } \\
\text { edge angle } \\
\text { (pre } \rightarrow \text { post) }\end{array}$ & $\begin{array}{l}\text { Medial clear } \\
\text { space } \\
\text { (pre } \rightarrow \text { post) }\end{array}$ & $\begin{array}{l}\text { Tönnis grade } \\
\text { (pre } \rightarrow \text { post) }\end{array}$ \\
\hline $\begin{array}{l}\text { Mild dysplasia } \\
(N=19)\end{array}$ & $\begin{array}{l}11^{\circ} \rightarrow 5^{\circ} \\
(p<0.0001)\end{array}$ & $\begin{array}{l}21^{\circ} \rightarrow 31^{\circ} \\
(p<0.0001)\end{array}$ & $\begin{array}{l}11^{\circ} \rightarrow 42^{\circ} \\
(p<0.0001)\end{array}$ & $\begin{array}{l}11 \mathrm{~mm} \rightarrow 6 \mathrm{~mm} \\
(p<0.0001)\end{array}$ & $\begin{array}{l}0=13 \rightarrow 11 \\
1=5 \rightarrow 5 \\
2=1 \rightarrow 2 \\
3=0 \rightarrow 1\end{array}$ \\
\hline $\begin{array}{l}\text { More severe } \\
\text { dysplasia } \\
(N=163)\end{array}$ & $\begin{array}{l}25^{\circ} \rightarrow 12^{\circ} \\
(p<0.0001)\end{array}$ & $\begin{array}{l}5^{\circ} \rightarrow 23^{\circ} \\
(p<0.0001)\end{array}$ & $\begin{array}{l}2^{\circ} \rightarrow 28^{\circ} \\
(p<0.0001)\end{array}$ & $\begin{array}{l}14 \mathrm{~mm} \rightarrow 9 \mathrm{~mm} \\
(p<0.0001)\end{array}$ & $\begin{array}{l}0=136 \rightarrow 118 \\
1=23 \rightarrow 26 \\
2=3 \rightarrow 16 \\
3=1 \rightarrow 3\end{array}$ \\
\hline
\end{tabular}

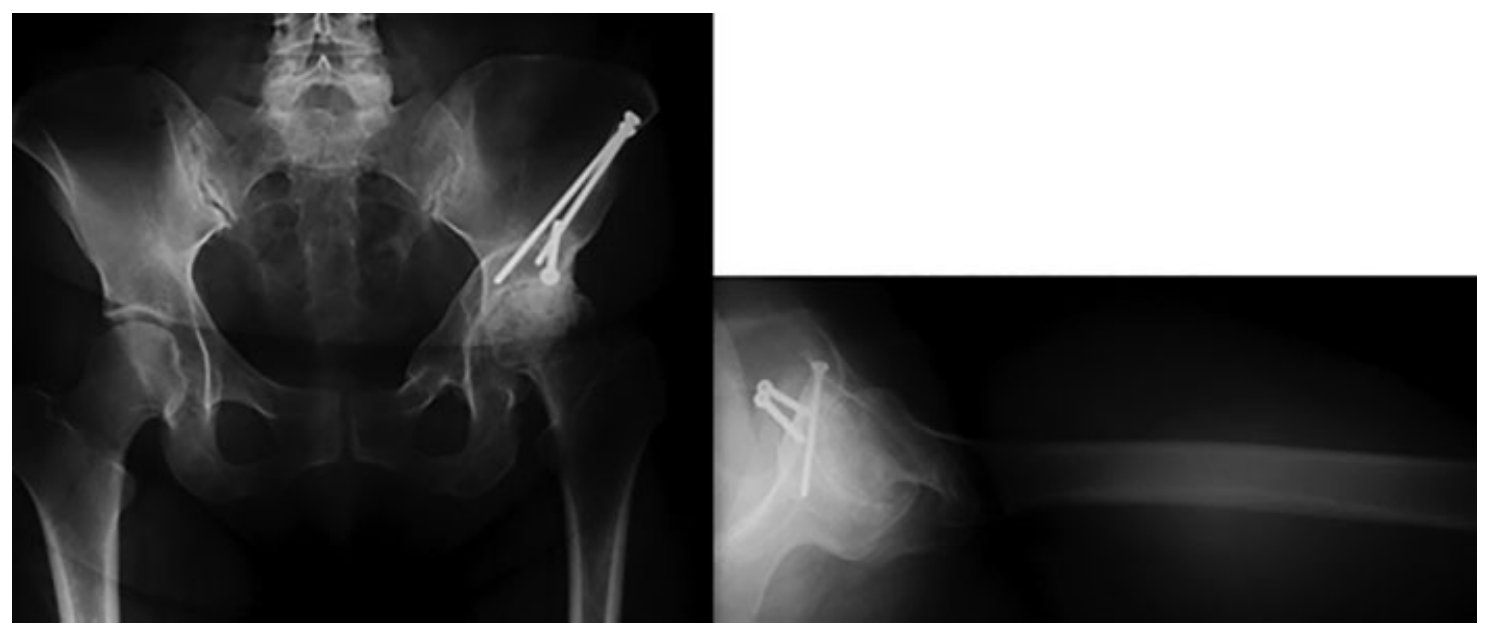

Fig. 1 AP and lateral radiographs show a patient in the MHD group that had Tönnis grade progression and underwent a THA 6 years after a PAO. AP, anterior-posterior; MHD, mild hip dysplasia; PAO, periacetabular osteotomy; THA, total hip arthroplasty. 


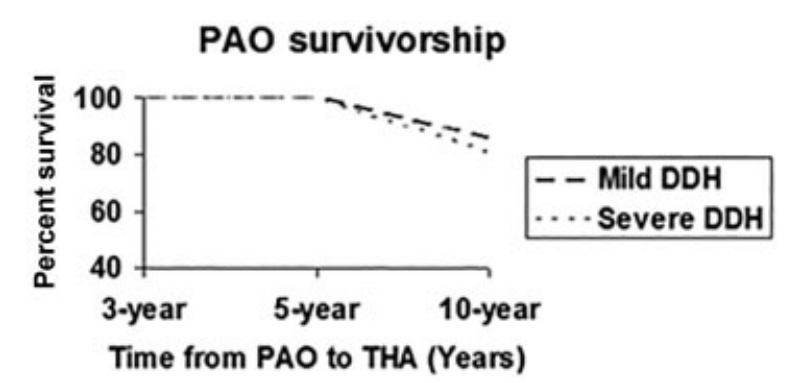

Fig. 2 Kaplan-Meier survivorship curves. DDH, developmental dysplasia of the hip; PAO, periacetabular osteotomy; THA, total hip arthroplasty.

MHD group. The corresponding rates for hips in the control group at 3, 5, and 10 years, respectively, were 99\% (95\% CI: [96.7, 100.0]), 95\% (95\% CI: [91.5, 99.6]), and 81\% (95\% CI: $[71.9,90.4])$.

\section{Discussion}

There continues to be debate whether patients with MHD should undergo PAO or hip arthroscopy. There is emerging data on the results of hip arthroscopy in this group, but the results of PAO are lacking. ${ }^{10}$ The purpose of this study is to present the results of PAO in this group in comparison to patients with more SHD. As such, this study should serve as a comparison for future studies looking at the results of hip arthroscopy in patients with MHD.

This study demonstrates that a PAO is a viable option in patients with MHD. The PAO leads to significant improvements in radiographic coverage of the femoral head and dramatic improvements in pain and function as assessed by the HHS that showed on average an excellent result at the last follow-up (92 points). Furthermore, there were no documented complications associated with the procedure indicating that the procedure, when done well, has an acceptable complication rate. Coinciding with this cohort, Zaltz et al reported a relatively low $5.9 \%$ major complication rate in periacetabular osteotomies. $^{23}$

There are several limitations to this study. First, we are not comparing PAO to hip arthroscopy for MHD. We are simply trying to set the stage so that future studies of hip arthroscopy used to treat MHD can be compared. Second, the number of MHD was small. This implies that a selection bias against PAO was likely present at our institution during the study period. Traditionally a PAO has not been recommended for this group of patients for the reasons described above, and several these may have been referred back for hip arthroscopy. Third, the decision to use measurements to define MHD was somewhat arbitrary and do not explain the entire pathomechanics associated with hip dysplasia and instability. It is well known that there are patients with what seem to be fairly normal radiographic measurements who display more symptoms of instability than others do, and hip dysplasia and instability may involve both static and dynamic features that cannot be defined by a numerical value on a radiograph. Finally, we are not recommending whether a PAO should or should not be done on all patients with MHD, we are simply reporting the results of PAO in this select group of patients and demonstrating that it is a reliable, safe, and effective in treating the structural disorder.

A previously published study on the results of hip arthroscopy for hip dysplasia demonstrated improvements in pain and function. Byrd and Jones reported on 48 hips with hip dysplasia treated arthroscopically and followed up between 12 and 60 months. ${ }^{24}$ The cohort included patients with both borderline hip dysplasia and those with more severe forms treated for several reasons. The average HHS in these groups was 83 and 77, respectively. Patients with labral pathology, chondral damage, and arthritis had average hip scores of 78, 75 , and 60 , respectively. Two hips required THA. Independent of the age of the patient, the average postoperative HHS in this group was below 90 . The results of PAO in the present series compares favorably to this published report. The postoperative clinical results in the present series were excellent with an average HHS of 92 points.

More recently, Domb et al ${ }^{10}$ have reported the results of a cohort of patients with MHD undergoing contemporary arthroscopic management. They used similar values for hip dysplasia as in our study (LCE between 18 and 25). The authors reported good to excellent results in $77 \%$ of 22 hips available for follow-up with three hips showing progression in arthritis grade within 24 months from surgery. Two patients required revision surgery. This paper shows that despite modern hip arthroscopic procedures and techniques that include labral repair and capsular plication, the results of hip arthroscopy in this group are decent at best and will likely get worse and seem to be below those reported here in this paper that has much longer follow-up.

Fukui et al similarly recently reported the results of 28 patients with mild-to-moderate hip dysplasia and concomitant femoroacetabular impingement (FAI) undergoing hip arthroscopy. ${ }^{25}$ This was a select group of patients from the practice of the senior author of that paper that had either labral repair or reconstruction, with all other patients with labral debridement excluded. All these patients had radiographic signs of hip dysplasia, with half of them having signs of acetabular retroversion and combined femoral head neck abnormalities. In this group, likely considered best case scenario, where the labrum was either repaired or reconstructed, there were nine re-operations (two revision arthroscopy, two PAO, and five THA). The modified HHS at the final follow-up of those 21 hips that survived either PAO or THA was 82 , also below those reported in this study.

The decision to proceed with either PAO or hip arthroscopy is not easy. It would seem intuitive that the results should be better when the structural deformity and the instability are addressed, and the labrum and cartilage can be protected. The downside of the PAO lies in its complexity, its potential complications that are thought to be higher than those occurring after hip arthroscopy, and the fact that some patients are not willing to undergo a major operation to correct the problem, especially in the athletic population. ${ }^{26,27}$ In addition, one previous study showed that a PAO may also be successful after a failure of hip arthroscopy 
and may support the use of hip arthroscopy in this setting with the rationale that the procedure does not "burn bridges." ${ }^{28}$ There are other studies on their way that may actually show that the results of PAO after hip arthroscopy may not be as successful as a matched cohort of patients undergoing PAO without previous surgery. We believe that modern arthroscopic treatment with labral repair and capsular plication may play a role in the treatment of some patients with milder forms of hip dysplasia. However, it is hard to know who is a good candidate for this procedure, and some patients will fail arthroscopic treatment alone. It should not be used as a sole treatment of hip dysplasia in patients with SHD, and we believe should not be used either in patients who clearly have symptoms associated with instability. In addition, the clinical results of the procedure are fair to good at best as described by Byrd, Domb, and Fukui et al, and the results of such intervention should be communicated to the patient.

In this series, there was significant improvement in radiographic femoral head coverage. The correction however is not easy. Many of these patients have mild anterolateral undercoverage of the femoral head. The correction should include a degree of retroversion to correct the anterior under coverage while also providing improvement in lateral coverage by rotating the fragment. Overcorrection of the acetabular fragment will lead to iatrogenic impingement. We did not particularly see this complication in this series, but did note increased ACE angles in some hips done earlier in the series. ${ }^{29}$ Intraoperative range of motion testing is mandatory to detect impingement and if necessary, re-orientation of the fragment, femoral neck junction osteochondroplasty, or anterior-inferior iliac spine trimming should be performed to correct both intra- and extra-articular impingement.

A 20-year follow-up study on the PAO demonstrated a survivorship of $60 \%{ }^{30}$ At 30 years, the data suggest that the PAO may actually save about one third of the hips from clinical deterioration or THA. The results of these papers are promising especially because the series included patients with neurogenic hip dysplasia and surgical techniques that we feel have been refined with an understanding of iatrogenic impingement and addressing intra-articular pathology. Patients in this study should represent a best-case scenario for a series of PAO, as the majority of patients were young and had Tönnis arthritis grade 0 or 1 . At 10 years, our curve showed that the survivorship was $89 \%$ with only one patient requiring a THA at 6 years for progression radiographic grading of arthritis. With the current understanding of the PAO and its limitations in patients with more severe forms of hip arthritis, that patient today would unlikely be a candidate for PAO in our hands. The second THA in the MHD group was at 15 years and was performed due to hip pain that was later found to originate from her lumbar spine. The rest of the patients had excellent clinical and radiographic results and have neither had progression in hip arthritis nor any other additional surgical procedure beyond removal of symptomatic hardware.

Based on the present series, PAO is an appropriate surgical option for treating patients with MHD. In this setting, the operation has an acceptable complication rate, provides improvements in the structural abnormality, and has excellent clinical results comparable to patients with more SHD. Patients with MHD in this study did well with a PAO, and the results were maintained into midterm. Recently, arthroscopy has been reported as an alternative to PAO, but only shortterm results have been reported with less favorable pain and functional results. This study however does not compare and cannot recommend against hip arthroscopy in select patients with mild hip dysplasia, but is a useful comparison in the absence of published data comparing the two procedures. A randomized trial comparing PAO and hip arthroscopy in patients with mild hip dysplasia would be ideal to shed further light on the subject.

\section{Conflict of Interest}

None.

\section{References}

1 Harris WH. Etiology of osteoarthritis of the hip. Clin Orthop Relat Res 1986;(213):20-33

2 Murphy SB, Kijewski PK, Millis MB, Harless A. Acetabular dysplasia in the adolescent and young adult. Clin Orthop Relat Res 1990; (261):214-223

3 Solomon L. Patterns of osteoarthritis of the hip. J Bone Joint Surg Br 1976;58(02):176-183

4 Stulberg SD, Harris WH. Acetabular dysplasia and development of osteoarthritis of hip. In: Harris WH, ed. The Hip. Proceedings of the Second Open Scientific Meeting of the Hip Society. St. Louis, MO: Mosby; 1974:82-93

5 Tönnis D, Legal H, Graft R, eds. Congenital Dysplasia and Dislocation of the Hip in Children and Adults. Berlin, Germany: SpringerVerlag; 1987

6 Murphy SB, Ganz R, Müller ME. The prognosis in untreated dysplasia of the hip. A study of radiographic factors that predict the outcome. J Bone Joint Surg Am 1995;77(07):985-989

7 Lequesne M; de SEZE. False profile of the pelvis. A new radiographic incidence for the study of the hip. Its use in dysplasias and different coxopathies [In French]. Rev Rhum Mal Osteoartic 1961; 28:643-652

8 Tönnis D. Eine neue form der huftfannenschwenkkung durch dreifachosteostomie zur ermoglichung spoterer hufprothesenversorgung. Orthop Praxis 1979;15:1003-1005

9 Wiberg G. Studies on dysplastic acetabulae and congenital subluxation of the hip joint. Acta Orthop Scand Suppl 1939;58:1-132

10 Domb BG, Stake CE, Lindner D, El-Bitar Y, Jackson TJ. Arthroscopic capsular plication and labral preservation in borderline hip dysplasia: two-year clinical outcomes of a surgical approach to a challenging problem. Am J Sports Med 2013;41(11):2591-2598

11 Bogunovic L, Gottlieb M, Pashos G, Baca G, Clohisy JC. Why do hip arthroscopy procedures fail? Clin Orthop Relat Res 2013;471(08): 2523-2529

12 Clohisy JC, Nepple JJ, Larson CM, Zaltz I, Millis M; Academic Network of Conservation Hip Outcome Research (ANCHOR) Members. Persistent structural disease is the most common cause of repeat hip preservation surgery. Clin Orthop Relat Res 2013;471 (12):3788-3794

13 Jackson TJ, Watson J, LaReau JM, Domb BG. Periacetabular osteotomy and arthroscopic labral repair after failed hip arthroscopy due to iatrogenic aggravation of hip dysplasia. Knee Surg Sports Traumatol Arthrosc 2014;22(04):911-914

14 Matsuda DK, Khatod M. Rapidly progressive osteoarthritis after arthroscopic labral repair in patients with hip dysplasia. Arthroscopy 2012;28(11):1738-1743 
15 van Bergayk AB, Garbuz DS. Quality of life and sports-specific outcomes after Bernese periacetabular osteotomy. J Bone Joint Surg Br 2002;84(03):339-343

16 Clohisy JC, Barrett SE, Gordon JE, Delgado ED, Schoenecker PL. Periacetabular osteotomy for the treatment of severe acetabular dysplasia. J Bone Joint Surg Am 2005;87(02):254-259

17 Crockarell J Jr, Trousdale RT, Cabanela ME, Berry DJ. Early experience and results with the periacetabular osteotomy. The Mayo Clinic experience. Clin Orthop Relat Res 1999;(363): 45-53

18 Kralj M, Mavcic B, Antolic V, Iglic A, Kralj-Iglic V. The Bernese periacetabular osteotomy: Clinical, radiographic, and mechanical 7-15 year follow-up of 26 hips. Acta Orthop 2005;76(06): 833-840

19 Siebenrock KA, Leunig M, Ganz R. Periacetabular osteotomy: the Bernese experience. Instr Course Lect 2001;50:239-245

20 Trousdale RT, Cabanela ME. Lessons learned after more than 250 periacetabular osteotomies. Acta Orthop Scand 2003;74(02): 119-126

21 Trumble SJ, Mayo KA, Mast JW. The periacetabular osteotomy. Minimum 2 year followup in more than 100 hips. Clin Orthop Relat Res 1999;(363):54-63

22 Tönnis D. Normal values of the hip joint for the evaluation of Xrays in children and adults. Clin Orthop Relat Res 1976;(119): $39-47$
23 Zaltz I, Baca G, Kim YJ, et al. Complications associated with the periacetabular osteotomy: a prospective multicenter study. J Bone Joint Surg Am 2014;96(23):1967-1974

24 Byrd JW, Jones KS. Hip arthroscopy in the presence of dysplasia. Arthroscopy 2003;19(10):1055-1060

25 Fukui K, Trindade CA, Briggs KK, Philippon MJ. Arthroscopy of the hip for patients with mild to moderate developmental dysplasia of the hip and femoroacetabular impingement: outcomes following hip arthroscopy for treatment of chondrolabral damage. Bone Joint J 2015;97-B(10):1316-1321

26 Davey JP, Santore RF. Complications of periacetabular osteotomy. Clin Orthop Relat Res 1999;(363):33-37

27 Hussell JG, Rodriguez JA, Ganz R. Technical complications of the Bernese periacetabular osteotomy. Clin Orthop Relat Res 1999; (363):81-92

28 Kain MSH, Novais EN, Vallim C, Millis MB, Kim YJ. Periacetabular osteotomy after failed hip arthroscopy for labral tears in patients with acetabular dysplasia. J Bone Joint Surg Am 2011;93(Suppl 2):57-61

29 Ziebarth K, Balakumar J, Domayer S, Kim YJ, Millis MB. Bernese periacetabular osteotomy in males: is there an increased risk of femoroacetabular impingement (FAI) after Bernese periacetabular osteotomy? Clin Orthop Relat Res 2011;469(02):447-453

30 Steppacher SD, Tannast M, Ganz R, Siebenrock KA. Mean 20-year followup of Bernese periacetabular osteotomy. Clin Orthop Relat Res 2008;466(07):1633-1644 